phate be taken, and one or two drops of a solution of urate of soda, containing $.648 \mathrm{grm}$. of uric acid, in $100 \mathrm{c}$. c., be added, a permanent precipitate of uratc of zinc will result ; and, although this precipitate is certainly soluble, it is very slightly so in neutral or slightly acid solutions. It is soluble in caustic alkali and in free acid.

If 10 c. c. of the ahove solution of urate of soda be placed in a urea estimation apparatus, with sodium hypobromite, it will, at $60^{\circ} \mathrm{Fahr}$. and 30 in barometer, yield $8.5 \mathrm{c}$. c. of nitrogen gas. If $10 \mathrm{c.}$. of such a solution be added to $90 \mathrm{c}$. c. of water, and precipitated with a strong solution of zinc sulphate, the precipitate washed with water saturated with zinc urate and then filter and precipitate placed in a urea estimation apparatus, with sufficient sodium hypobromite of full strength, it will yield $8 \mathrm{c}$. c. nitrogen at the above temperature and pressure.

To apply these facts to the estimation of uric acid in urine, it is suffcient to take 300 or $4 \mathrm{co} \mathrm{c.} \mathrm{c.} \mathrm{of} \mathrm{the} \mathrm{urine} \mathrm{and} \mathrm{add} \mathrm{a} \mathrm{few} \mathrm{drops} \mathrm{of} \mathrm{strong}$ solution of caustic soda, enough to render it decidedly alkaline; allow the precipitate of phosphates to subside, and pour off 100 c. c. of the clear solution; add to this clear solution about $4 \mathrm{c}$. $\mathrm{c}$. of a solution of zinc sulphate, $\mathbf{I}$ in 3 strength. The solution of zinc sulphate renders blue litmus red, and it must be added to the urine until a drop of the mixture on blue litmus gives a slightly red circle. If the solution be too acid, a drop or two of caustic solution will render it neutral. The precipitate is thrown on a filter, and washed with a saturated solution of zinc urate. This solution is made by adding a little zinc sulphate to distilled water, and then adding urate of soda until a permanent precipitate remains ; this is allowed to subside, and the solution is used for washing until all urea and ammonia are removed. The drained precipilate is folded in the filter, and placed, with $50 \mathrm{c}$. c. of hypobromite of sodium of full strength, in an urea estimation apparatus, and agitated carefully. The gas crolved will be an indication of the amount of uric acid present in 100 c. c. of the urine. The evolution of gas does not commence immediately, and there is sufficient time to insert the stopper and note the number at which the water stands in the gas-tube. If a reaction begin immediately, the precipitate has nct been sufficiently washed. The reaction is complete at the end of half an hour, without the application of heat. On many occasions, when the urine precipitate was mixed with the hypobromite, a blue colour appeared, and again disappeared, and at first this was supposed to be due to a substance other than uric acid; but, in repeated experiments with urate of zinc, made from pure white crystals of uric acid, the same blue colour was obscrved. This blue is not indigo. A quantity of pure urate of soda was precipitated with zinc sulphate, and washed; the precipitate mixed with distilled water, and hypobromite of sodium added drop by cirop; the colour appeared, and deepened to a dark blue. Tartaric acid was then added, when the colour clisappeared, and would not return when the solution was rendered alkaline; nor on further addition of hypobromile. The zinc precipitate can be dissolved in caustic soda solution, diluted, and used with boiling Fehling's solution, in the manner proposed by Pavy.

Effect of the Presence of Ammoniacal Salts on the Precipitate.-Ten c. c. of the standard solution of urate of soda were added to $90 \mathrm{c}$. c. of water, and I grain of ammonium chloride added. The mixture was then precipitated as usual with zinc sulphate, filtered, washed, and placed in urea apparatus; the gas yielded equalled $8 \mathrm{c}$. c., indicating that the presence of ammoniacal salts is not detrimental, as the whole of the ammonia can be removed by washing.

Hippurate of zinc is soluble in water, and the other nitrogenous constituents of urine, if precipitated with the uric acid, are in too small amounts to sensibly affect the result.

With a filter pump, the precipitation, and washing, and evolution of gas, may all be completed in one and a half hours; and, of course, several experiments may proceed at one time.

Alihough this process will not give a mathematically accurate $\epsilon$ stimation of uric acid, it is one which, with ordinary care, will give very approximate results; and, although it may be urged that the solubility of zinc urate may be greater in urine than in water, yet the results of a -number of experiments will always be comparable with each other. It may be possible that, under special circumstances other substances may be precipitated, and may interfere with the estimation, yet, in normal urine, the sources of error are small, and a series of estimations have given very interesting results; moreover, there are many drugs which will not themselves affect the composition of the precipitate, and therefore are no bar to accuracy.

In describing his new process for uric acid estimation, Pavy suggests that, if his results be confirmed, the normal amount excreted by a healthy adult is much higher than has been hitherto supposed. The results obtained by the above-described process confirm this observation; and in a series of experiments, carried on with but slight inter- mission for three months, the total uric acid excreted by one individual, in apparent health, was never less than Io grains, and seldom less than 12 grains, per twenty-four hours; while many apparently trivial circum. stances increased the amount largely. The results were occasionally confirmed by decomposing the zinc precipitate with dilute sulphuric acid, drying and weighing the resulting crystals. It was found that, when hydrochloric acid was used to dissolve the zinc urate, therc was much less tendency for the uric acid to separate. The results of a series of observations, and the effect of the administration of various drugs in a healthy individual, will be given in a future paper in detail; but it may be stated here that any results, based on the estimation of urine voided at any given period of the day, are utterly fallacious; and, fur. ther, an estimation, based on the analysis of the urine of any one period of twenty-four hours, is also apt to be misleading.

\section{SOME PRACTICAL POINTS IN THE TREATMENT OF HÆMOPTYSIS.*}

\section{BY JAMES M. WILLIAMSON, M.D.EDiN., Ventnor.}

IN bringing forward, in a very brief manner, some practical points in relation to this question, I will, for the convenience of the first part of my object, divide cases of hæmoptysis into three kinds : first, the slight ; second, the copious; and third, what may be termed the explosive.

In the slight form, the basis of the sputum is composed of mucus which is stained more or less deeply with blood, the bleeding ressel being of small size. The most successful remedy for this form is ergot.

In the second or copiones variety, the expectoration consists of pure blood, the quantity of which may vary up to a very large amount; and the bleeding ceases gradually until the attack is over. Here again the most successful remedy is ergot, and indeed it is in this kind of hæmoptysis that ergot is especially efficacious. In order to prove efficient in hxmoptysis, however, ergot must be given boldly. One teaspoonful of the liquid extract is a suitable dose, and it may be ordered every half-hour, hour, or two hours, according to the urgency of the case. If it is doing good, it is a mistake to leave it off before the sputa are bloodless, although the intervals between the doses will be lengthened as the hxmorrhage abates. In a few of these cases, ergot will fail; not in many, but now and then. If seven or eight doses be ineffectual, it is best to abandon it. The next remedy worthy of confidence is gallic acid, which it is necessary to give freely, in doses of fifteen to twenty grains, at intervals the same as in the case of ergot. Should there be tedious delay in the final clearing up of small traces of blood from the sputa, an acid mixture with quinine is usually effectual; or, if very obstinate, ipecacuanha, in twenty-minim doses of the wine pushed to slight nausea, will generally remove them.

In the third or cxplosive variety of hæmoptysis, the attacks are profuse, sudden in their onset, all at once ceasing, often for many hours, then abruptly bursting out again. There is no gradual subsidence. The lesion is probably a rupture of some aneurysmal sac in the wall of a cavity. Now it is in these cases that ergot is hardly ever of much use. In my experience, the best remedy is turpentine internally, with cold applications over the chest. Three half-drachm doses of oil of turpentine may be given, half an hour apart; or, it care be taken to follow it with castor-oil, even more than three. When the turpentine is left off, it is well to follow up closely with a mixture of gallic and aromatic sulphuric acids, sulphate of magnesia, and quinine. It is particularly in this type of case that digitalis is often useful for calming vascular excitement. As the patients often make blood very rapidly, the free use of aperients ought to be enjoined.

Nothing would be easier than to quote a long string of remedies for hrmoptysis, but my present object is to leave prominently on the mind one or two that are to be relied upon, and to indicate their spheres of usefulness. Nor is it necessary to dwell on certain instructions which apply to all forms of blood-spitting. Constipation must not go unrelieved, and is best treated by salines. A quick pulse must be steadied by digitalis, of which perhaps the most handy form is the digitaline granule of Homolle and Quevenne. Cough is to be soothed; the simpler the mode of accomplishing this the better, but it must be done; and nothing answers better for this than a chloroform pad laid over the sternum.

Speaking in a general way, and not alluding to hæmoptysis of cardiac origin, I hold that we should keep before our minds the advisability of stopping all blood-spitting in phthisis without delay. To this rule, perhaps, there are two exceptions. The first is trivial. It is

* Introduction to a discussion on the treatment of hæmoptysis, at the January meeting of the Isle of Wight District of the Southern Branch. 
that dirty-red, slimy, bad smelling, never abundant expectoration which hysterical women with phthisis often exhibit at the bottoms of their spittoons; this may be left to itself. The other exception is a serious one; it comprises those forms of hæmoptysis, usually copious and angry, occurring in advanced and very chronic cases where there is a considerable amount of fibroid induration. In such patients, notable dyspnca on exertion has for a long time past been a prominent symptom, and respiration has been maintained by a very small extent of lung-substance. These cases are open to a special danger-that of fatal embolism in the right chambers of the heart or the pulmonary artery. Not uncommonly, the course followed is for the bleeding gradually to abate in quantity, remaining, nevertheless, of the same angry red; then urgent dyspnœa suddenly sets in, and death takes place within forty-eight hours. These are cases calling for extremely careful treatment. Can it be right, where only a small surface is available for respiratory function, to contract those few vessels with ergot? Or can it be good practice to pass styptic medicines into a patient's circulation when his cachectic state, his low vitality, and perhaps some febrile movement, render him especially liable to the formation of thrombi ? It is wisest to limit ourselves to external applications, chloroform-pads, dry cupping, sinapisms at a distance or other derivative treatment, with appropriate general management.

Perhaps I may be allowed to conclude with two cautions, commonplace they may seem, but both of them the outcome of bedside experi ence. One is, to have some responsible person in attendance night and day, on all cases of severe bleeding, till the attack has completely passed away. Death in hæmoptysis is generally sudden, and it is very appalling to discover too late the consequences of omitting this precaution. The other is, to decline positively to examine a patient's chest while there is any hæmoptysis. Irrespectively of the danger of the process, an opinion arrived at by auscultating a chest during or immediately after a bleeding is not a reliable one.

\section{NOTE ON A CASE OF PSEUDO-HYPERTROPHIC PARALYSIS (?): RECOVERY.}

BY H. DONKIN, M.B., F.R.C.P.,

Senior Assistant-Physician, Westminster Hospital; Physician to the East London Hospital for Children.

ON the Igth of November last (1881), I saw a boy six years old among my out-patients at Westminster Hospital, whose mother brought him on account of his having been unaccountably "falling about" for a week, and being quite unatle to run or walk fast. She stated that he had been "slow and inactive" for about five or six weeks previously, and that she had noticed that his calves and buttocks seemed larger than before. These facts were given spontaneously, not elicited by questions.

The child's previous medical history included the occurrence of some inflammation of the eyes at birth, measles, and whooping-cough. At three years old, he was observed to be excitable and passionate, this state lasting some months; but he had no "fits" of any kind. The only other child in the family died at the age of six months with diarrhcea. There was no evidence of parental syphilis. The mother had had two miscarriages; and the history of the father, who is dead, was obscure. Subsequent inquiry failed to find the trace of any affection like pseudo-hypertrophic paralysis in the mother's family.

The boy stood with his legs wide apart, straddled and swung from side to side as he slowly walked, fell down on attempting to walk quickly, and was unable to raise himself from the sitting posture without pressing on one of his knees, and previously turning round and sitting on all-fours. There was protrusion of the abdomen, and a noteworthy degree of lordosis. On stripping him, it was obvious to me and the students who were present that his calves and buttocks were remarkably large for a boy of his size. The skin, especially over the lower part of the body, was markedly mottled; and, after repeated trials, I failed to elicit the knee-jerk, except perhaps very slightly in the left leg. Knowing, however, how erroneous it often is to attach any value to the absence of this latter phenomenon in children, and the general want of knowledge about the importance of this symptom at all, I mention it only because, on the recovery of the boy, which is the excuse for this record, the knee-jerk was elicited in both legs with as much ease as could be expected in the case of a rather excitable child. There was not any obvious enlargement of the muscles of the upper extremity. But I was able on that day to make but an imperfect examination, and unfortunately postponed both the measurement of the girth of his calves and all further methods of inquiry. Owing to an unavoidable absence, I did not see the case again till January 7 th, more than six weeks after the first visit. Then, to my surprise, beyond some waddling in walking, I noticed no symptoms of loss of power. The boy could run with ease, and raise himself from the ground in the ordinary manner, even without placing his hands on the floor. The calves still appeared to me to be somewhat large; but the mother, again spontaneously, observed that both the calves and buttocks had "got quite small again". There was no mottling of skin. On the r4th, I saw him again. He still waddled slightly in walking; and I again failed, as I had also on the 7 th, to produce any knee-jerk after careful trial. The mother believed the child to be now quite well. On the 21st, I could detect nothing abnormal about the child or his movements, with the exception of the knee-jerk symptom. I saw him again on February roth, when I did not examine him; snd for the last time on body normal; the gait in no way altered; running performed with perfect ease; and the knee-jerk produced with tolerable readiness.

The treatment I ordered from the first was complete rest; the boy having previously been exercised by the mother, believing, as she did, that he was careless. For medicine, as he looked somewhat weakly and pale, although quite cheerful, I gave him our mixture of iron and nux vomica. This he discontinued taking on February roth.

I am well aware that this is but an imperfect report of the case, anci that the diagnosis may be open to question. Still there is enough to show that we may have to look out at least for a set of symptoms simullating the disease about which so much has been written and so little is known. And there would seem to be no reason in nature why there should not be some temporary affection of the nerve-centres (even if this disease be regarded as connected with changes in the cord) giving rise to transient symptoms, just as we have the same possibility hinted to us in the occurrence of other forms of recovering paralysis.

I would disclaim any very probable connection between the drug. treatment and the result, believing that, to whatever the symptoms were due, rest must be far more reasonably credited with their disap. pearance.

\section{STRANGULATED UMBILICAL HERNIA AFTER PARTURITION : OPERATION : RECOVERY.*}

\section{BY HERBERT BRACEY, M.R.C.S.Eng., Birmingham.}

MRS. L., aged 4I, who had had twelve children, "and one miscarriage, had suffered from bronchitis for some years. She was confined on January $30 t h, 1881$, at nearly full time. Labour was natural. The child was alive, and fully developed. The patient was suffering from a severe attack of bronchitis at the time of parturition. Great care was taken in applying the binder securely, as the abdomen was very flabby, and when she coughed the abdominal muscles seemed to give way, as if they had no power of resistance. There was no appearance of any tendency to umbilical hernia, nor had she ever suffered from one. $I$ left her at 9 P.M., and was called to her again at 4 A.Mr. the follow. ing day, when I found her suffering intense pain, and discovered an umbilical hernia, about the size of an orange, very tense, and nearly black. With difficulty this was reduced, a pad was placed over the ring, and a plate and several napkins over that, and the binder firmly applied.

The next day, February Ist, I applied a pad made on purpose, and secured it by strapping, and all seemed to be going on well; but on the next day, February 2 nd, the third day from the confinement, the cough being still very violent, the hernia was again protruded. The coumptoms were very urgent, intense pain and great distress; vcmiting, which nothing checked; anxious expression of countenance; with small feeble pulse and cold clammy skin. Reduction was again triec, but failed; and after consultation with Mr. Bartleet, it was decided that operative measures were imperative. The patient was put under chloroform by my assistant Mr. Holdsworth; and, with Mr. Bartleet's kind assistance, I made an incision in the median line, over what appeared to be the neck of the sac, and divided the very thin layers of fascia on a director. On reaching the sac, the ring was found to be very tight. An incision was made, without opening the sac, in a direction immediately upwards, but still reduction was difficult; when suddenly the sac burst, and its contents, which appeared to be partly omentum and partly bowel, were readily returned. The rent in the sac was carefully not examined ; but the whole thing was quickly pinched up, and the skin brought together by silk sutures; and a pad, plaster, and bandage were applied. The patient was at once relicved, and no vomiting recurred.

It is unnecessary to record the daily notes of temperature and pulse. It is sufficient to say that the progress of the case was one of uninter-

* Read before the Midland Medical Society. 